\title{
Protein Nanopore-Based Discrimination between Selected Neutral Amino Acids from Polypeptides
}

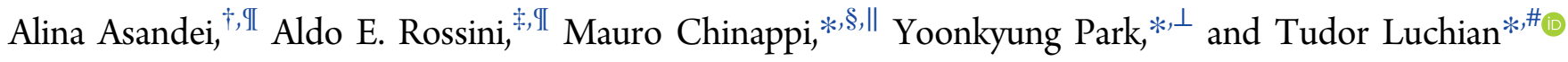 \\ ${ }^{\dagger}$ Interdisciplinary Research Department and ${ }^{\#}$ Department of Physics, Alexandru I. Cuza University, Iasi 700506, Romania \\ ${ }^{\ddagger}$ Department of Basic and Applied Science for Engineering, Sapienza University of Rome, Via A. Scarpa14, 00161 Rome, Italy \\ ${ }^{\S}$ Department of Industrial Engineering, University of Rome Tor Vergata, Via del Politecnico 1, 00133 Rome, Italy \\ "Center for Life Nano Science@Sapienza, Istituto Italiano di Tecnologia, Via Regina Elena 291, 00161 Rome, Italy \\ ${ }^{\perp}$ Department of Biomedical Science and Research Center for Proteinaceous Materials (RCPM), Chosun University, Gwangju, Korea
}

\section{Supporting Information}
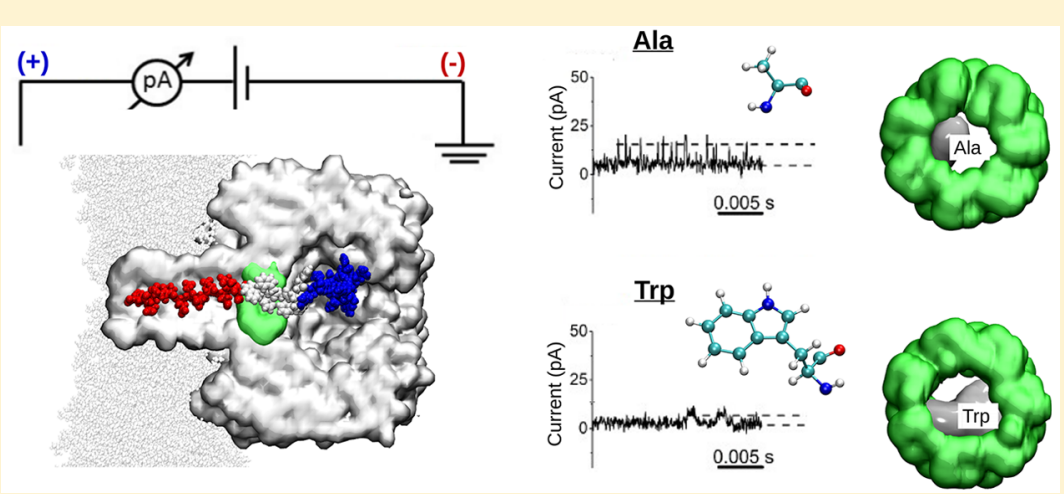

ABSTRACT: Nanopore probing of biological polymers has the potential to achieve single-molecule sequencing at low cost, high throughput, portability, and minimal sample preparation and apparatus. In this article, we explore the possibility of discrimination between neutral amino acid residues from the primary structure of 30 amino acids long, engineered peptides, through the analysis of single-molecule ionic current fluctuations accompanying their slowed-down translocation across the wild type $\alpha$-hemolysin $(\alpha$ $\mathrm{HL}$ ) nanopore, and molecular dynamics simulations. We found that the transient presence inside the $\alpha$-HL of alanine or tryptophan residues from the primary sequence of engineered peptides results in distinct features of the ionic current fluctuation pattern associated with the peptide reversibly blocking the nanopore. We propose that $\alpha$-HL sensitivity to the molecular exclusion at the most constricted region mediates ionic current blockade events correlated with the volumes that are occluded by at least three alanine or tryptophan residues, and provides the specificity needed to discriminate between groups of neutral amino acids. Further, we find that the pattern of current fluctuations depends on the orientation of the threaded amino acid residues, suggestive of a conformational anisotropy of the ensemble of conformations of the peptide on the restricted nanopore region, related to its relative axial orientation inside the nanopore.

\section{INTRODUCTION}

One of the major advances in today's nanotechnology stems from the advent of nanopores, either protein-, solid state-, or hybrid-based, which allowed unprecedented probing of molecular interactions at the nanometer scale and submillisecond time resolution. ${ }^{1-3}$ The working principle of the approach uses a potential difference to drive the target ion or molecular complex through the nanopore. As this occurs, a corresponding volume of solvent gets displaced, the electrical resistance of the nanopore most often increases, and this leads to reversible changes in the ionic electrical current measured across the nanopore. The statistical and volumetric analysis of such blockade events is later used to infer information about the physical and chemical properties of the studied analyte.
An acclaimed nano(bio)technology application of biological nanopores is represented by identification of each successive nucleotide in a DNA or RNA strand, which catalyzed the nascent technologies allowing the rapid and low-cost sequencing applications for biomedical or other life sciences research. $^{4-10}$

A rich application of nanopore technology for proteomics is to detect and conformationally analyze, and potentially sequence, peptides or proteins. To date, however, nanopores have proven remarkable successful mostly for the first two objectives. $^{11-19}$ The results of all-atom molecular dynamics

Received: September 7, 2017

Revised: October 29, 2017

Published: November 27, 2017 
simulations regarding the feasibility of using graphene nanopores for protein sequencing have revealed that the nanopore transport of the peptides produces stepwise modulations of the nanopore ionic current, which were found correlated with the type of amino acids present in the nanopore. ${ }^{20}$ The successful protein sequencing is hampered by mainly two factors: (i) unlike nucleic acid-based polymers, the rich variety of topologies, namely, secondary, tertiary, and quaternary structures of proteins, overcomplicates the interpretation of the induced current blockade events across the nanopore in unequivocal terms of primary structure details; (ii) the heterogeneous charge distribution on a protein poses difficulties in controlling the protein dynamics alongside electric field lines inside the nanopore. In addition, at least two critical challenges are to be met by the nanopore technology, before it can successfully be used in protein sequencing. On one hand, there is a need to provide single amino acid resolution. Note, for example, that the most constricted region of one of the commonly used biological nanopores (e.g., $\alpha$-hemolysin from Staphylococcus aureus, $\alpha$ HL), located between Met-113 and Lys-147 and Glu-111, is $\sim 0.6 \mathrm{~nm}$ in length and $1.4 \mathrm{~nm}$ in diameter, ${ }^{21,22}$ setting a theoretical spatial resolution of $\sim 1.6$ amino acids. As an additional difficulty, previous studies regarding the ssDNA trafficking across the Mycobacterium smegmatis porin A (MspA), which has a thinner constriction domain $(\sim 0.5 \mathrm{~nm})$ and a smaller in diameter constriction domain $(\sim 1.2 \mathrm{~nm})$ than the $\alpha$ $\mathrm{HL},{ }^{23}$ demonstrated that the length of DNA probed is actually longer than the length of the pore's constriction. ${ }^{24,25}$ On the other hand, another fundamental issue is to control the electrodiffusive journey of a polymer (ssDNA, peptide, protein) past the constriction region in the nanopore, to successfully fingerprint individual monomers-induced blockade events from the noisy background, and identify the primary structure of the polymer. To address this last issue, various approaches have been envisaged, exploiting the influence of physical and chemical properties of the solvent on analyte transit across nanopores, the interaction of analytes with specific antibodies, using a pressure-voltage biased pore or entropic trapping of biomolecules near nanopores, or altering the nanopore's physical properties and controlling its surface charge. ${ }^{26-34}$

Despite all recognized drawbacks, the interest for nanoporebased protein sequencing to achieve unparalleled sensitivity and throughput remains central, as the existing methods commonly employed for this task, namely, mass spectrometry and Edman degradation, still pose critical challenges, despite their maturity in use. Concisely, these methods are slow, consuming time, resources, and reagents. ${ }^{35,36}$ By mitigating some of the experimental challenges highlighted above, recent studies have demonstrated that nanopores can be successfully used to inspect the primary structure of a protein. ${ }^{37-41}$

Here, we recorded ion current measurements fluctuations across the wild type $\alpha$-HL nanopore interacting with 30-aminoacid-long engineered peptides, and demonstrate the proof-ofconcept enabling primary structure exploration of polypeptides, via discrimination between selected neutral amino acid residues.

\section{MATERIALS AND METHODS}

Electrophysiology. Lipid membrane bilayers were obtained from 1,2 diphytanoyl-sn-glycero-3-phosphocholine (Avanti Polar Lipids, US) dissolved in $n$-pentane (HPLC grade, Sigma-Aldrich, Germany) using the Montal-Muller technique. ${ }^{42}$ Briefly, the dissolved lipid formed a stable solventless bilayer structure across an $\sim 120 \mu \mathrm{m}$ in diameter orifice punctured on a $25-\mu \mathrm{m}$-thick Teflon film (Goodfellow, Malvern, MA), pretreated with 1:10 hexadecane/pentane (HPLCgrade, Sigma-Aldrich, Germany), that separates the cis (grounded) and trans chambers of the recording cell. All of the experiments were performed at a room temperature of $\sim 23{ }^{\circ} \mathrm{C}$ and both chambers of the bilayer cell were filled with a $2 \mathrm{M} \mathrm{KCl}$ solution, buffered in $10 \mathrm{mM}$ HEPES at $\mathrm{pH}=7$. To achieve the insertion of a single nanopore channel into the lipid bilayer, small volumes $(\sim 0.5-2 \mu \mathrm{L})$ of $\alpha$ hemolysin $(\alpha$-HL) protein (Sigma-Aldrich, Germany) were added from a monomeric stock solution made in $0.5 \mathrm{M} \mathrm{KCl}$ to the cis compartment. Once a stable $\alpha$-HL protein nanopore was inserted in a supporting lipid membrane, the peptides denoted by Pe1 (Ac- $(\mathrm{R})_{12}-$ $\left.(\mathrm{A})_{6}-(\mathrm{E})_{12}-\mathrm{NH}_{2}\right), \operatorname{Pe} 3\left(\mathrm{Ac}-(\mathrm{R})_{12}-(\mathrm{W})_{6}-(\mathrm{E})_{12}-\mathrm{NH}_{2}\right)$, or Pe5 (Ac$\left.(\mathrm{R})_{12}-(\mathrm{A})_{3}-(\mathrm{W})_{3}-(\mathrm{E})_{12}-\mathrm{NH}_{2}\right)$ (synthesized and purified by Schafer-N ApS, (Copenhagen, Denmark)) were added in the trans chamber of the bilayer cell at a bulk concentration of $20 \mu \mathrm{M}$ from a $1 \mathrm{mM}$ stock solution made in distilled water. Ionic current fluctuations through the nanopore, reflecting the unimolecular reversible interactions between the peptides and the $\alpha$-HL protein, were recorded at holding potentials $(\Delta V)$ varying from $\pm 20 \mathrm{mV}$ to $\pm 90 \mathrm{mV}$. The reversible peptide-nanopore interactions were detected and amplified using an Axopatch 200B (Molecular Devices, USA) instrument. Data acquisition was performed with a NI PCI 6221, 16-bit acquisition board (National Instruments, USA) at a sampling frequency of 50 $\mathrm{kHz}$, and were low-pass filtered at a corner frequency $\left(f_{\mathrm{c}}\right)$ of $10 \mathrm{kHz}$, within the graphical programming environment LabVIEW 8.20 (National Instruments, USA). To protect the experimental setup from electromagnetic and mechanic interference, the bilayer chamber was housed in a Faraday cage (Warner Instruments, USA), and placed on the top of a vibration-free platform (BenchMate 2210, Warner Instruments, USA). Numerical analysis and data graphing were done with the help of the Origin 6 (Origin Lab, USA) and pClamp 6.03 (Molecular Devices, USA) software. The statistical analysis on the frequency and duration of the peptide-induced current fluctuations through a single $\alpha$-HL protein were analyzed within the statistics of exponentially distributed events, as previously described. ${ }^{43}$ The current values were derived from the amplitude histograms by fitting the distributions to Gaussian functions. At least three independent experiments were carried out in order to arrive at the numerical estimates reported herein.

Molecular Dynamics Simulation. All-atom Molecular Dynamics (MD) simulations were performed using the NAMD software. ${ }^{44}$ The CHARMM36 force field ${ }^{45}$ was employed to model lipid, protein, and TIP3P water molecules. ${ }^{46}$ NBFIX corrections were applied for ions. ${ }^{47}$

Setup and Equilibration. The membrane-pore system has been assembled using a protocol similar to previously presented ones. ${ }^{22,48,49}$ In brief, the system was assembled starting from the $\alpha$-HL crystal structure PDB_ID: 7AHL ${ }^{21}$ downloaded from the OPM database. ${ }^{50}$ The POPC membrane, the water molecules, and the ions for neutralizing the system were added using VMD. ${ }^{51}$ Then, the system is minimized and a $60 \mathrm{ps}$ NVT simulation (time step $0.2 \mathrm{fs}$ ) was run with external forces applied to water to avoid that water molecules enter the membrane and pore. Lipid heads have been constrained to their initial position by means of harmonic springs acting on the phosphorus atoms (spring constant $k=\frac{1 \mathrm{kcal}}{\mathrm{mol} \AA^{2}}$ ). A second equilibration run ( $1 \mathrm{~ns}$ NPT flexible cell, time step $1 \mathrm{fs}$ ) was performed to compact the membrane. During this run the lipid heads were free to move. The third, and last, equilibration step consists of a NPT constant area simulation ( $2 \mathrm{~ns}$, time step $2 \mathrm{fs}$ ) where all the atoms are unconstrained. The resulting periodic box after the equilibration has the following size: $L_{x}=12.75 \mathrm{~nm}, L_{y}=12.71 \mathrm{~nm}$, and $L_{z}=18.00 \mathrm{~nm}$, and the total number of atoms is 302000 . Initial configurations of peptides are generated by using the PEPFOLD server ${ }^{52}$ and then separately equilibrated in a triperiodic water box. The box dimensions after a constant area NPT equilibration ( $5 \mathrm{~ns}$, time step $2 \mathrm{fs}$ ) are $L_{x}=4.83$ $\mathrm{nm}, L_{y}=4.83 \mathrm{~nm}$, and $L_{z}=4.83 \mathrm{~nm}$. Then, the two systems were merged, ions $(2 \mathrm{M} \mathrm{KCl})$ were added using VMD, and a short NPT equilibration is performed ( $2 \mathrm{~ns}$, constant area NPT). The resulting 


$$
\text { a) } A c-(R)_{12}-(X)-(E)_{12}-N H_{2} \quad X=\left\{\begin{array}{c}
A_{6} \\
W_{6} \\
A_{3} W_{3}
\end{array}\right\}
$$

b)

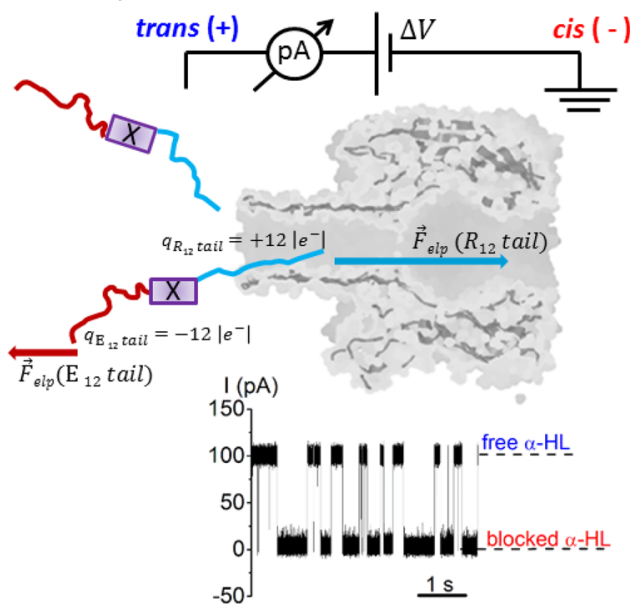

c)

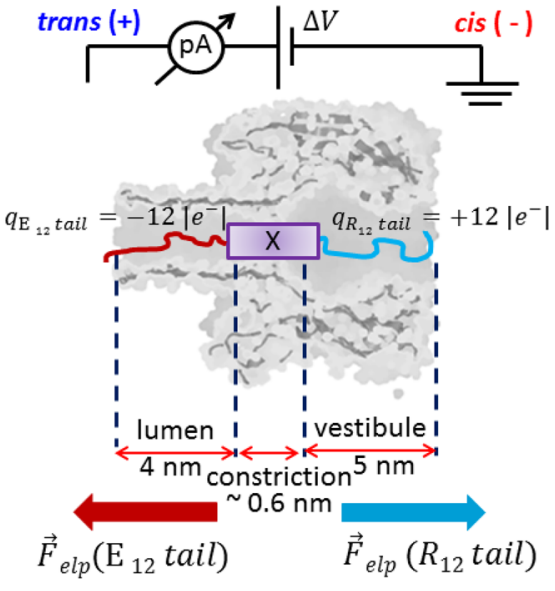

Figure 1. Schematic illustration of the approach to detect single peptides interaction with the $\alpha$-HL nanopore. Once the nanopore is formed in a supporting lipid membrane, the peptide (designated in panel a by Ac- $(\mathrm{R})_{12}-(\mathrm{X})-(\mathrm{E})_{12}-\mathrm{NH}_{2}$ ) is added to the trans chamber of the recording apparatus. We used different sets of peptides, engineered to contain in the middle ' $\mathrm{X}$ ' domain either six Ala, six Trp, or a combination of three Ala and three Trp residues, flanked by the oppositely charged tails at neutral $\mathrm{pH}$, each containing $12 \mathrm{Arg}$ and Glu residues. Depending of the sign of the applied transmembrane voltage on the trans side, the peptide is funneled to the lumen region of the nanopore with the Arg- (at positive potentials, as shown here) or Glu-containing tail head on (at negative potentials). As shown in panel $b$, the main driving force for peptide capture by the nanopore is the electric force acting on the Arg-containing tail of the peptide $\left(\vec{F}_{\text {elp }}\left(\mathrm{R}_{12}\right.\right.$ tail $\left.)\right)$. This force is larger than the oppositely oriented electric force acting on the Glu-containing tail $\left(\vec{F}_{\text {elp }}\left(\mathrm{E}_{12}\right.\right.$ tail $\left.)\right)$, since the electric field is more intense in the immediate vicinity of the nanopore and inside it. In the excerpted trace in panel $\mathrm{b}$, it is illustrated that as the peptide interacts with the nanopore ("blocked $\alpha$-HL"), reversible changes in the ionic current through the open nanopore ("free $\alpha$-HL") ensue. In its metastable state, whereby the net result of electric forces acting oppositely at the peptide termini is approximately nil (panel c), the middle section of the peptide is located near the $\alpha$-HL's constriction region (see also text). Marked distinctly are the lumen, constriction, and, respectively, vestibule domains of the nanopore.

box has dimensions $L_{x}=12.75 \mathrm{~nm}, L_{y}=12.71 \mathrm{~nm}$, and $L_{z}=18.62 \mathrm{~nm}$, and the overall number of atom is $\sim 303000$.

Dedicated steered molecular dynamics simulations were employed to bring the peptides at the pore's lumen entrance (trans side) and then inside the nanopore. In particular, the peptide N-terminus (Arg tail) was placed at $\sim 1.5 \mathrm{~nm}$ from the $\alpha$-HL's trans entrance and then pulled inside the nanopore using a constant velocity Steered Molecular Dynamics simulation.

Ionic Flux Measurement. Nine representative configurations along the translocation path have been selected. In the first three, the constriction is occupied by Arg, in the second three by the central residue (Ala or Trp), and in the last three by Glu. These configurations have been used as initial conditions for nonequilibrium runs where a homogeneous and constant electric field $\mathbf{E}=\left(0,0, E_{z}\right)$ acting along the $z$ direction is applied. This is equivalent to the application of a constant voltage $\Delta V=E_{z} L_{z}{ }^{53}$

As usual for nonequilibrium all-atom MD simulations, to reduce the statistical errors, the applied voltage is larger than the experimental one; in particular, we used $\Delta V=1 \mathrm{~V}$. The duration of production runs spans from 75 to $120 \mathrm{~ns}$. Snapshots are saved every $20 \mathrm{ps}$ and average currents are estimated as in ref 7 after discarding a $\sim 40$ ns transient. During the production runs, the lipid heads were constrained via a harmonic spring acting on the phosphorus atoms, spring constant $k=$ $\frac{1 \mathrm{kcal}}{\mathrm{mol} \AA^{2}}$.

\section{RESULTS AND DISCUSSION}

In our simplified approach, to improve the readout of ion current blockade signature of amino acids through volumetric measurements in single-molecule electrophysiology experi- ments, we focused on residues with marked difference in their physical size, namely, Ala $\left(\sim 100 \AA^{3}\right)$ and $\operatorname{Trp}\left(\sim 239 \AA^{3}\right)$.

The peptides were engineered such that the primary sequence section harboring the residues to be distinguished between (Ala, Trp, and a combination between the two, dubbed by ' $X$ ', Figure 1) were flanked by segments of oppositely charged amino acids (Figure 1). By using this nonconventional approach, the applied transmembrane potential enhances the polypeptide capture rate by the $\alpha$-HL nanopore, and simultaneously increases the peptide's residence time in the nanopore. ${ }^{54-56}$

Figure 2 demonstrates that by increasing the applied potential magnitude, both the capture rate of the peptides used herein (quantified as the inverse of the average $\tau_{\text {on }}$ values) and their average residence time inside the nanopore $\left(\tau_{\text {off }}\right)$ increase correspondingly.

As we show in Supporting Information Figure S1, the statistical distributions of interevents $\left(\tau_{\text {on }}\right)$ and blockade-event $\left(\tau_{\text {off }}\right)$ durations of data displayed in Figure 2, calculated at a given transmembrane potential, were found to be exponential. Furthermore, owing to the oppositely charged termini of the peptides used, the induced current blockades were observed at both positive and negative applied potentials, with the peptide added to the trans side alone (see Figure S2). This is suggestive of a chain of events during which, depending on its sign, the electric field near the nanopore opening stemming from the transmembrane potential $(+$ or $-\Delta V)$ orients the polypeptide to enter the pore entrance with the Arg or Glu tail head on, and 
a)

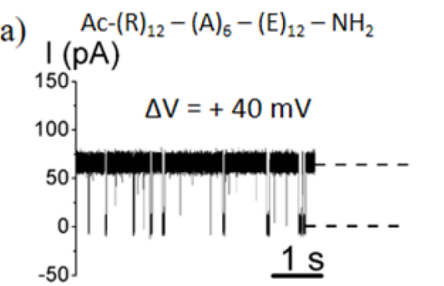

d)

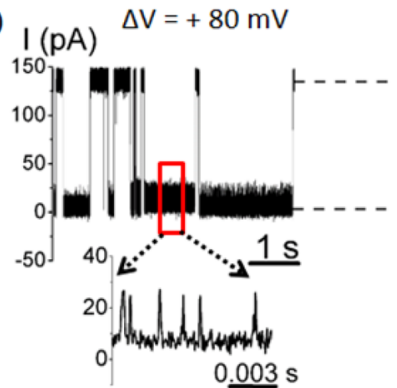

g)

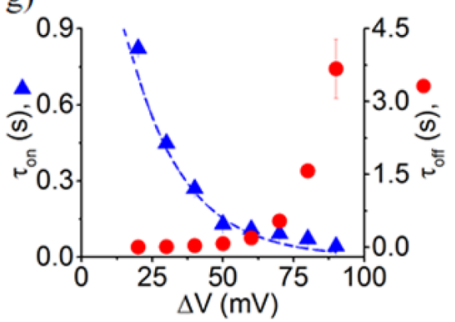

b)

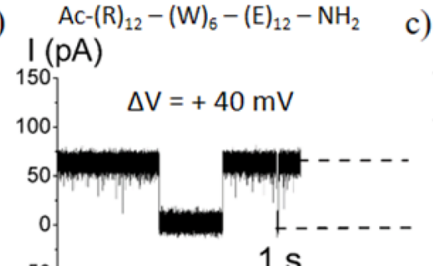

e)

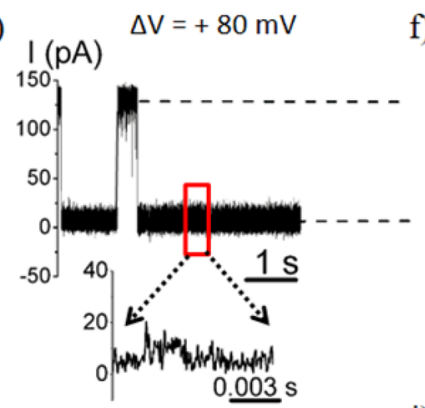

h)

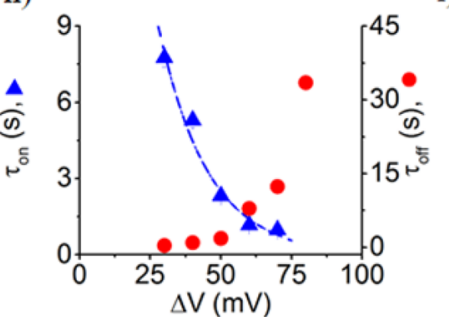

i)
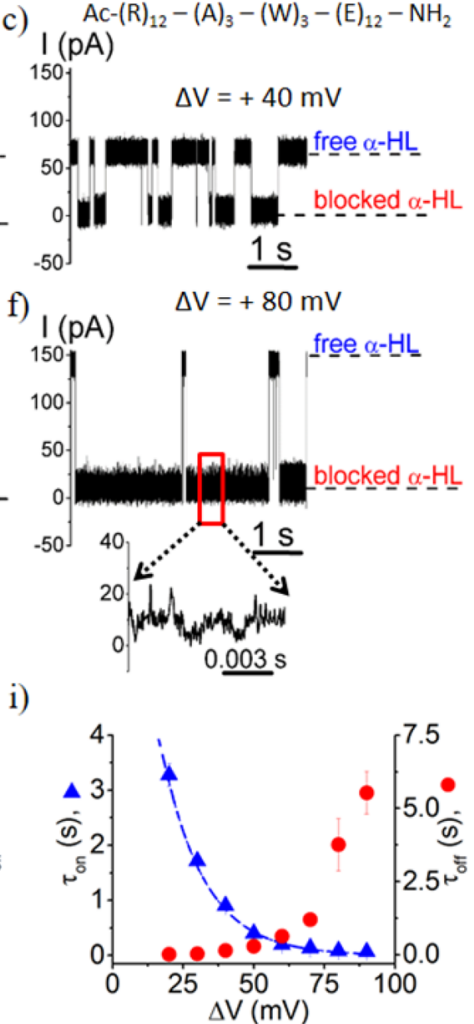

Figure 2. Selected traces displaying the reversible blockades of the ion current through an open $\alpha$-HL pore due to interactions with the peptides. Addition of either peptide construct $\left(\mathrm{Pe} 1 \mathrm{Ac}-(\mathrm{R})_{12}-(\mathrm{A})_{6}-(\mathrm{E})_{12}-\mathrm{NH}_{2}\right.$, panel a; Pe3 Ac- $(\mathrm{R})_{12}-(\mathrm{W})_{6}-(\mathrm{E})_{12}-\mathrm{NH}_{2}$, panel b; or Pe5 Ac- $(\mathrm{R})_{12}-(\mathrm{A})_{3}-$ $(\mathrm{W})_{3}-(\mathrm{E})_{12}-\mathrm{NH}_{2}$, panel c) to the trans side of the membrane at a bulk concentration of $20 \mu \mathrm{M}$, in an electrolyte containing $2 \mathrm{M} \mathrm{KCl}, 10 \mathrm{mM}$ HEPES, $\mathrm{pH}=7$, leads to nanopore blockades, seen as downward events from the current measured across the free $\alpha$-HL. As the applied potential increases from $\Delta V=+40 \mathrm{mV}$ (panels a, $\mathrm{b}$, and c) to $+80 \mathrm{mV}$ (panels e, $\mathrm{f}$, and $\mathrm{g}$ ), the average duration of the blockade events $\left(\tau_{\text {off }}\right)$ increases, whereas the average of time intervals measured in between successive peptide capture events by the nanopore $\left(\tau_{\text {on }}\right)$ decreases. The representative zoomed-in segments in panels $\mathrm{d}-\mathrm{f}$ illustrate the ionic current fluctuations through the $\alpha$-HL during a metastable capture of the corresponding peptides (see text). In panels $\mathrm{g}$, h, and $\mathrm{i}$ are shown the applied potential dependence of average values of the inter-event time intervals $\left(\tau_{\mathrm{on}}\right.$; "free $\alpha$-HL") and the blockade-event durations $\left(\tau_{\text {off; }}\right.$ "blocked $\alpha$-HL"), for the three sets of peptides used. The average capture times were fit to a single exponential $\left(\tau_{\text {on }}=\right.$ $\left.A \mathrm{e}^{-\Delta V / \Delta V_{0}}\right)$, and the corresponding parameters were as follows: $A=1.98 \pm 0.54 \mathrm{~s}, \Delta V_{0}=19.51 \pm 2.39 \mathrm{mV}(\operatorname{Pe} 1) ; A=45.55 \pm 11.79 \mathrm{~s}, \Delta V_{0}=17.22$ $\pm 1.7 \mathrm{mV}(\mathrm{Pe} 3)$; and $A=11.95 \pm 2.65 \mathrm{~s}, \Delta V_{0}=15 \pm 0.96 \mathrm{mV}(\mathrm{Pe} 5)$.

facilitates its capture by the nanopore's $\beta$-barrel. Considering the negatively charged lumen entrance at neutral $\mathrm{pH}$ (bare charge of $\sim-7\left|e^{-}\right|$, the peptide association at the lumen entrance is augmented via electrostatic attractive interactions when the positively charged Arg moiety moves toward the nanopore, ${ }^{55}$ so that the subsequent experiments were focused for the case of positively applied transmembrane potentials.

A useful strategy used herein was built around the fact that, once inside the nanopore, the oppositely charged peptide gets trapped in the free-energy minimum. This metastable state, which is reflected by the peptide dwelling longer inside the $\alpha$ $\mathrm{HL}$ as $\Delta V$ increases (Figure 2, panels $\mathrm{g}-\mathrm{i}$ ), is determined by a configuration in which the peptide gets positioned symmetrically relative to the middle (constriction) region of the nanopore, ${ }^{54}$ implying that the number of oppositely charged amino acids reaching to the $\alpha$-HL's lumen and vestibule domains, respectively, and the values of the oppositely oriented electrostatic forces acting on the ends of the peptide are largely similar.

The existence of the metastable state is due to the combination of the nonhomogeneous electrical field associated with the presence of the nanopore and the strong dipole of the molecule. $^{54,56}$ The applied potential induces a nonhomogeneous electrical field that is more intense inside the nanopore.
Outside the nanopore, molecules align their dipoles along the field lines converging into the nanopore. As the molecule enters the nanopore, it experiences a gradually increasing importing force, due to the larger electrical field. When the other charged tail also engages the nanopore, an opposite couple of forces acting on the two tails sets in, and the analyte becomes stably trapped approximatively in the middle of the nanopore, where the two forces balance. From this equilibrium position, further movements of the polypeptide toward the cis or trans side (panel d) result in a net electrical force that tend to drive the peptide back to the equilibrium position, i.e., a "nanopore tweezer" effect appears.

The trapping mechanism of the peptide by the nanopore can be conveniently described in terms of free energy profiles. As a first approximation, the free-energy associated with the peptide translocation can be expressed as the sum of two independent terms, $G_{\text {ext }}$ due to the applied voltage, and $G_{s}$, associated with entropic penalty of the confinement into the pore. ${ }^{56}$ For symmetric systems, both $G_{\text {ext }}$ and $G_{s}$ are symmetric and, consequently, the free-energy minimum corresponds to a conformation with the central region of the translocating peptide near the nanopore's center. The $\alpha$-HL nanopore is asymmetric; hence both $G_{\text {ext }}$ and $G_{s}$ contributions are asymmetric as well, and consequently, the metastable 
equilibrium point of the trapped peptide can get shifted toward the $\alpha$-HL's vestibule or $\beta$-barrel. Electrical potential maps reported previously, ${ }^{22}$ suggest that for a peptide configuration like the one reported in Figure 4, panel a, the force acting on the negative residues should be larger than the one acting on the positive ones. Hence, the peptide should move toward the nanopore's $\beta$-barrel. On the other hand, since the $\alpha$-HL's vestibule is larger than the $\beta$-barrel, the entropic contribution $G_{\mathrm{s}}$ is asymmetric, and it is expected to move the equilibrium point toward the vestibule, as the peptide passage from the vestibule to the $\beta$-barrel comes at an entropic cost. Hence, although a definitive answer is not available, we expect that the free-energy minimum corresponding to a peptide as engineered herein, trapped inside the $\alpha-\mathrm{HL}$, is correlated with the central residues of the peptide present at the $\alpha$-HL's constriction region.

Thus, from geometric considerations, and refereeing to a metastable peptide inside the $\alpha$-HL, the nanopore's constriction region is largely populated by the peptide's middle domain residues (section ' $\mathrm{X}$ ' in the primary structure, Figure 1), which become the dominant contribution to the ensuing current blockade amplitude changes. The $\alpha$-HL-peptide interactions at the nanopore's smallest nanovolume increase the sensitivity of the targeted amino acid readout, based on volumetric measurements through single-molecule electrophysiology.

To set a lower limit on the spatial resolution discrimination of these amino acids, and knowing that the $\sim 0.6 \mathrm{~nm}$ in length $\alpha$-HL's constriction region may harbor $\sim 1.6$ amino acids at a time (vide supra), we considered that a residue from the middle region of a peptide occluding transiently the $\alpha$-HL's constriction domain, as well as the adjacent ones flanking the constriction, most likely alter the recorded ionic current through the convolving effects of thermal position fluctuations. ${ }^{25}$ Thus, we hypothesized that the distribution of current amplitude fluctuations associated with the movement of the metastable peptide inside the nanopore is correlated with the volumes of at least three residues located in the peptide's middle section, presented successively on the $\alpha$-HL's constriction region.

In this line of argument, the prolonged transit of the peptide across the nanopore, associated with its metastable state, provided an ideal opportunity to fingerprint the distinct set of amino acids from its central region as they occlude the nanopore's most constricted region, through exploring the nature of peptide-induced conductance fluctuations of the $\alpha$ $\mathrm{HL}$ (Figure 2, panels $\mathrm{d}-\mathrm{f}$, zoomed-in traces in insets, and Figure 3).

As shown in Figure 3, the current block through the nanopore induced by a residing peptide, displays randomly occurring fluctuations between distinct blockade substates, marked as represented (\#, \$, and \&). We hypothesized (vide supra) that such events are most likely consistent with the reversible motion of the middle region of the peptide through the constriction domain of the nanopore, under the stalling electric force exerted at the ends of the peptide. In this scenario, each blockade fluctuation reflects a group of $\sim 3$ amino acids (either Ala or Trp, for the case of $\mathrm{Pe} 1$ or Pe3, respectively), occluding the nanovolume of the $\alpha$-HL's constriction region.

It may not be excluded that the ionic current fluctuations illustrated in Figure 3 do not reflect entirely the interactions of central (Ala or Trp) residues from the peptides with the $\alpha$-HL's constriction region, but contain contributions from the peptide's termini-located, Arg or Glu residues $-\alpha$-HL inter-
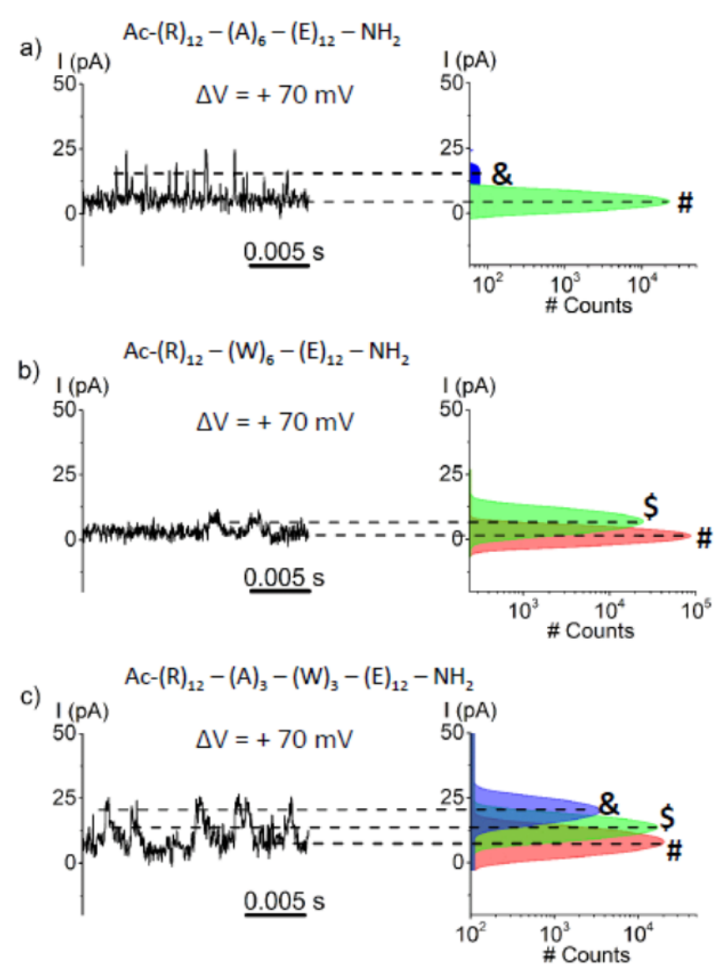

Figure 3. Detecting amino acids in a peptide from ion current fluctuations across the nanopore. Representative current recordings through a single $\alpha$-HL pore showing the transient pore blockades by a trapped Pe1 peptide (Ac- $(\mathrm{R})_{12}-(\mathrm{A})_{6}-(\mathrm{E})_{12}-\mathrm{NH}_{2}$ ) (panel a), Pe3 peptide $\left(A c-(R)_{12}-(W)_{6}-(E)_{12}-\mathrm{NH}_{2}\right)$ (panel b), or Pe5 peptide (Ac$\left.(\mathrm{R})_{12}-(\mathrm{A})_{3}-(\mathrm{W})_{3}-(\mathrm{E})_{12}-\mathrm{NH}_{2}\right)$ (panel $\left.\mathrm{c}\right)$, added in the trans side at a bulk concentration of $20 \mu \mathrm{M}$, in an electrolyte containing $2 \mathrm{M} \mathrm{KCl}, 10$ $\mathrm{mM}$ HEPES, $\mathrm{pH}=7$, and an applied transmembrane potential $\Delta V=$ $+70 \mathrm{mV}$. Due to the oppositely oriented electric forces acting at the termini of the peptide while it resides inside the nanopore, the peptide kinetics inside the pore slowed down sufficiently enough, so that the distinct blockade events induced by peptide movement along the constriction region of the nanopore are visible. Adjacent to each trace, which shows zoomed-in fluctuation of the ion current measured across the nanopore while a peptide resides inside (corresponding to the "blocked $\alpha$-HL" state), is the all-points histogram, showing the amplitude distribution of the sub-blockade events distinct from the fully blocked substate ('\#'), marked distinctly with ' $\&$ ' and ' $\$$ ', respectively.

actions, occurring as the peptide exits the nanopore through either the trans or cis side. Although entirely possible, the probability of catching such events in action is in fact very low, especially at the temporal resolution used herein, due to the speed of a translocating peptide through the nanopore. We reiterate that the metastability of the peptide trapped inside the $\alpha$-HL pore is determined by the net values of the electrostatic forces acting oppositely on both ends of the polypeptide, which should be close to zero (vide supra). In a scenario where during its passage across the nanopore, either the $\mathrm{N}$ - and $\mathrm{C}$-terminus of the peptide reaches the $\alpha$-HL's constriction region, the charged residues from the peptide along the nanopore's axial direction become unevenly distributed, i.e., a net difference between the number of positive and negative residues inside the nanopore ensues. The result is that the electric forces acting at the termini of the nanopore-trapped peptide no longer resemble a tug of war, failing to stabilize the peptide inside the pore sufficiently, as to enable the accurate reading of peptide termini- $\alpha$-HL constriction region interactions. In 
Table 1. Main Parameters Characterizing the Blockade Amplitude and Duration of Sub-State Events Elicited by a Metastable Pe1 Ac- $(\mathrm{R})_{12}-(\mathrm{A})_{6}-(\mathrm{E})_{12}-\mathrm{NH}_{2}$ or Pe3 Ac- $(\mathrm{R})_{12}-(\mathrm{W})_{6}-(\mathrm{E})_{12}-\mathrm{NH}_{2}$ Peptide, Transiently Trapped Inside the $\alpha$-HL Nanopore

\begin{tabular}{lllll} 
& \multicolumn{2}{c}{ Pe1 Ac- $(\mathrm{R})_{12}-(\mathrm{A})_{6}-(\mathrm{E})_{12}-\mathrm{NH}_{2}$} & \multicolumn{1}{c}{ Pe3 Ac- $(\mathrm{R})_{12}-(\mathrm{W})_{6}-(\mathrm{E})_{12}-\mathrm{NH}_{2}$} \\
$\Delta I_{\text {block }} / I_{\text {open }}$ & $0.83 \pm 6 \times 10^{-3}(\&)$ & $0.95 \pm 6 \times 10^{-3}(\#)$ & $0.93 \pm 4 \times 10^{-3}(\$)$ & $0.98 \pm 9.1 \times 10^{-4}(\#)$ \\
$\tau$ & $2.4 \times 10^{-4} \pm 0.4 \times 10^{-4} \mathrm{~s}(\&)$ & $0.008 \pm 3.2 \times 10^{-4} \mathrm{~s}(\#)$ & $0.003 \pm 2.4 \times 10^{-4} \mathrm{~s}(\$)$ & $0.007 \pm 7.7 \times 10^{-4} \mathrm{~s}(\#)$ \\
\hline
\end{tabular}

a)

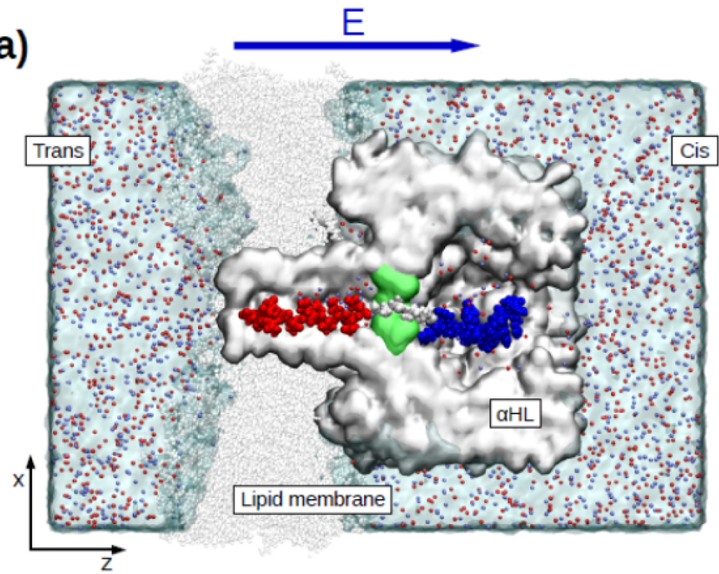

b)

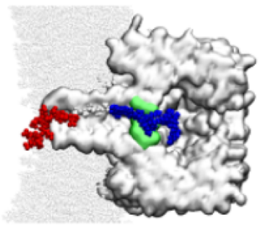

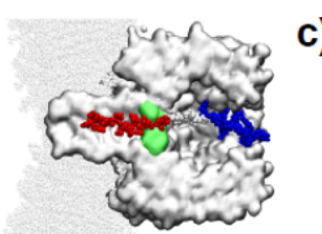

c)

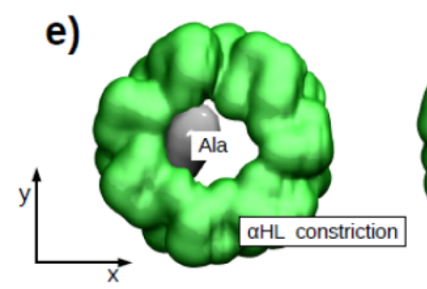

d)
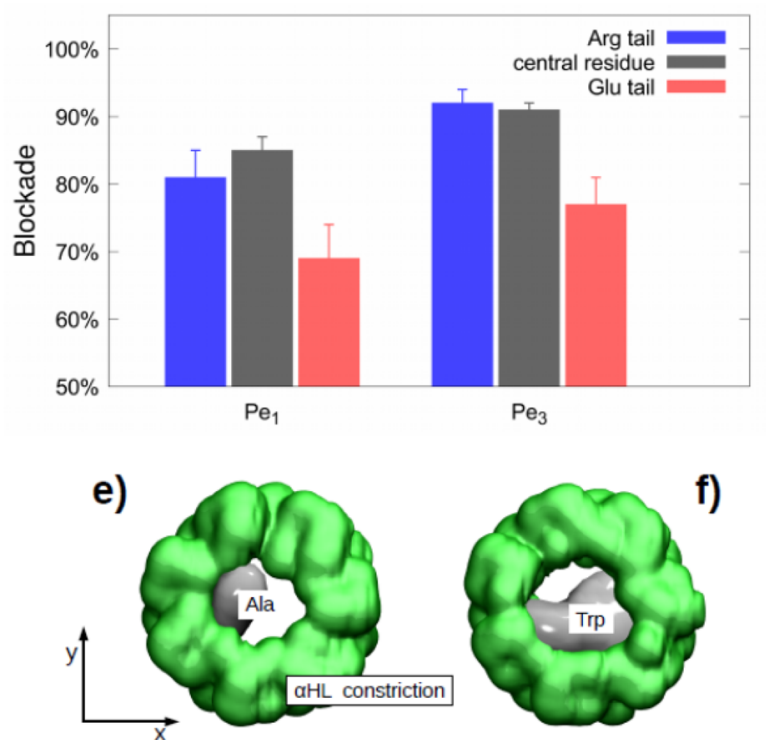

Figure 4. Molecular dynamics simulation. (a) The system is constituted by the $\alpha$-HL nanopore embedded in a lipid bilayer, the peptide, water, and ions ( $2 \mathrm{M} \mathrm{KCl}$ ). The $\alpha$-HL's constriction (Glu 111, Met 113, and Lys 147) is highlighted in green, while the peptide residues are colored according to their charge, i.e., the negative Glu tail is in red, the positive Arg tail is in blue, while the middle neutral domain (Ala of Trp) is in white. The peptide is imported in the pore from the barrel (trans) side with the positive Arg tail directed toward the vestibule (cis) entrance of the pore. Different configurations along the peptide translocation path have been selected and used as initial conditions for nonequilibrium runs where an electrical field $E$ is applied along the pore axis pointing from the trans to the cis side. Panels $\mathrm{a}, \mathrm{b}$, and $\mathrm{c}$ refer to Pel peptide. In panel a, the middle domain is in the pore constriction, while panels $\mathrm{b}$ and $\mathrm{c}$ show configurations where the constriction is occupied by one of the two charged tails. Panel $\mathrm{d}$ reports the mean current blockades resulting from nonequilibrium runs for both Pe1 and Pe3. The blue, gray, and red boxes refer to conditions where the constriction is occupied by the positive tail, the middle domain, and the negative tail, respectively. Snapshots of the constriction region (green) when occupied by Ala or Trp are shown in panels e and $\mathrm{f}$. The gray surfaces inside the constriction delimit the regions occupied by the peptide residues in a typical nonequilibrium run. It is apparent that Trp almost completely fills the pore constriction.

previous work with similar peptide constructs, and by using either a salt ${ }^{54}$ or $\mathrm{pH}$ gradient, ${ }^{55}$ we demonstrated that the unbalance of electric forces acting at the peptide termini while it resides inside the nanopore vigorously catalyzes the peptide exit from the nanopore, rendering events as those described in Figure 3 largely invisible.

To facilitate comparison between Pe1 and Pe3, the distribution of the blockades seen when a specific peptide interacted with the nanopore was assessed in terms of relative pore current blockade $\left(\frac{\Delta I_{\text {block }}}{I_{\text {open }}}\right.$, where $\Delta I_{\text {block }}=I_{\text {blocked }}-I_{\text {open }}$, $I_{\text {blocked }}$ is evaluated for either blockade substate '\#', '\$', or ' $\&$ ', and $I_{\text {open }}$ represents the ionic current measured through the free $\alpha$-HL), and the average duration of the events $(\tau)$, respectively, corresponding to the blockade substates '\#', '\$', or '\&' (Table 1).

As seen, the transient presence of the Ala- (Pe1) or Trpcontaining peptide ( $\mathrm{Pe} 3)$ inside the nanopore resulted in clearly distinct features of the fluctuation pattern associated with the peptide reversibly blocking the nanopore. Interestingly, the substates associated with the blockade events induced by the $\mathrm{Pe} 3$ peptide, containing the bulkier Trp, are more pronounced than those seen in the case of the Ala-containing peptide (Pe1) (compare $\frac{\Delta I_{\text {block }}}{I_{\text {open }}}$ for the most pronounced blockade events '\#' of both peptides, and the shallower ones (' $\$$ ' or ' $\&$ ', for the situation of $\mathrm{Pe} 3$ or $\mathrm{Pe} 1$, respectively). The analysis of these fluctuation patterns also reveal that the duration of the shallower substate events ('\$'-Pe3 or ' $\&$ '-Pe1) is larger for the case of the Trp-containing peptide ( $\mathrm{Pe} 3)$.

To interpret these amplitude fluctuations, we remind that the constriction region of the $\alpha$-HL $(\sim 0.6 \mathrm{~nm}$ in length and $\sim 1.4$ $\mathrm{nm}$ in diameter) has an estimated volume of $\sim 924 \AA^{3}$. In accord with the assertions mentioned above, we posit that the '\#' substate reflects the instance when a group of approximately three amino acids from the ' $\mathrm{X}$ ' domain of the peptides is being harbored transiently inside this narrowest volume. This is supported by the experimental observation that, due to the larger volume of Trp than Ala, the relative blockade amplitude of the "\#" substate is correspondingly larger for the Trpcontaining peptide (Pe3), as compared to the Ala-containing peptide (Pe1) (Table 1). Further than this, the reversible alterations seen in the amplitude of the '\#' blockade substate may reflect a convolving effect caused the partial exit from the constriction region of the nanopore of a group of approximately three amino acids from the ' $\mathrm{X}$ ' domain of the peptide, followed 
by the re-entry of another such group. In other words, the substates ' $\$$ ' (Pe3) and ' $\&$ ' (Pe1), respectively, reflect occupancy states of the nanopore while the constriction region contains a lesser number of amino acids as compared to the '\#' substate. This is supported the fact that the relative blockade amplitude of the ' $\$$ ' substate, corresponding to the Trpcontaining peptide $(\mathrm{Pe} 3)$, is slightly larger than that of the ' $\&$ ' substate, calculated for the Ala-containing peptide (Pe1) (Table 1).

To prove our hypothesis that the system presented here is capable of discrimination between groups of three distinct amino acids, from fluctuations in a single blockade event, we studied the interaction of another peptide construct (Pe5) (Ac$\left.(\mathrm{R})_{12}-(\mathrm{A})_{3}-(\mathrm{W})_{3}-(\mathrm{E})_{12}-\mathrm{NH}_{2}\right)$ with the nanopore. It is quite interesting that the analysis of the ionic current fluctuation patterns indicated three resolvable, distinct peaks, which for the deep ('\#') and, respectively, shallower events (' $\$$ ' and ' $\&$ ') showed relative blockade amplitudes $\left(\frac{\Delta I_{\text {block }}}{I_{\text {open }}}(\#)=0.94 \pm 1 \times\right.$ $10^{-3}, \frac{\Delta I_{\text {block }}}{I_{\text {open }}}(\$)=0.89 \pm 3 \times 10^{-3}$ and $\frac{\Delta I_{\text {block }}}{I_{\text {open }}}(\&)=0.84 \pm 2 \times$ $\left.10^{-3}\right)$, close to those measured in the case of Pe 3 and Pe 1 alone (see Table 1). This interesting observation was interpreted as putative evidence of our system to distinguish between groups of distinct amino acids, each containing either three Ala or Trp residues. Thus, it was reasoned that $\alpha$-HL nanopore sensitivity to the molecular volume at the constriction region mainly would promote the specificity necessary to discriminate between amino acids residues based solely on their volume.

To further interpret the experimentally observed current blockades in term of specific conformations of the peptide inside the nanopore, we performed all-atom Molecular Dynamics (MD) simulations for Pel and Pe3 peptides. The membrane-pore system, sketched in Figure 4, panel a, has been assembled and equilibrated using protocols similar to previously presented ones, ${ }^{22,48,49}$ also described in the Materials and Methods and Supporting Information.

After equilibration, the peptide is located at the pore trans entrance. Dedicated steered molecular dynamics simulations were employed to induce the peptide translocation. Nine representative configurations along the translocation path have been selected for each peptide. In the first three, the constriction is occupied by Arg (Figure 4, panel b), in the second three by the middle domain (Ala or Trp, Figure 4, panel a), and in the last three by Glu (Figure 4, panel c). These configurations have been used as initial conditions for nonequilibrium runs where a homogeneous and constant electric field $E_{z}$ acting along the $z$ direction is applied. This is equivalent to the application of a constant voltage $\Delta V=E_{z} L_{z}{ }^{53}$ As usual for nonequilibrium all-atom $\mathrm{MD}$ simulations, to reduce the statistical errors, the applied voltage is larger than the experimental one; in particular, we used $\Delta V=1 \mathrm{~V}$. In addition, we run a simulation without peptide to measure the open pore current. Mean current blockades are reported in Figure 4, panel d. For the case where the $\alpha$-HL's constriction is occupied by the peptide's middle neutral domain (gray bars), the $\mathrm{Pe} 3$ peptide results in a significantly deeper blockade $(0.91$ $\pm 0.015)$ with respect to the Pe1 peptide $(0.85 \pm 0.020)$. This phenomenon can be simply explained in terms of the different steric hindrance exerted by Trp and Ala at the nanopore's constriction region, as apparent in the Figure 4, panels e and f, where typical snapshots of the $\alpha$-HL's constriction occupied by either Ala and Trp resides are shown. The size difference between Ala and Trp affects also the ionic current through the nanopore, even when the constriction is being occupied by the positive arginine tail of the peptide (see blue bars in Figure 4, panel $d$ ). In such a scenario, the peptide's middle central domain is located in the $\alpha$-HL's $\beta$-barrel, as evidenced in Figure 4, panel $b$, for Pe1 and Figure S3, panel d, for Pe3. Instead, when the nanopore's constriction region is populated by the peptide's Glu negative tail, the ionic current blockade is much smaller. This can be ascribed to the fact that when Glu residues reside in the constriction region of the nanopore, the $\alpha$-HL's $\beta$ barrel is only partially occupied, resulting in a shallower blockage.

The presented approach has yet another untapped potential, namely, to drive the peptide with either the $\mathrm{N}$ - or C-terminal part inside the nanopore during the same experiment, by simply switching the sign of the applied potential (Figure S2). By exploiting this possibility, we found that a peptide entering the nanopore with the Glu-containing tail head, in otherwise similar experimental conditions as above except for the sign of the applied potential, gave rise to a pattern of current fluctuations strongly dependent on the orientation of the threaded amino acid residues (Figure S4). This is suggestive of a conformational anisotropy of the ensemble of conformations of the peptide on the restricted nanopore region, depending on its relative axial orientation to the nanopore, and we plan to investigate the molecular basis of this asymmetry and applications for amino acid discrimination further. A similar phenomenon has already been reported for the ssDNA translocation through the $\alpha$-HL nanopore. ${ }^{57}$ Related to this, in another study, it was also suggested that distribution of blockade events entailed by the passage of a peptide through a nanopore may also depend strongly on the configuration of the molecular termini of the peptide ( $\mathrm{N}$ - or $\mathrm{C}$-terminus) relative to the nanopore. ${ }^{37}$ At this moment, a clear explanation of data shown in Figure S4 is further complicated by the fact that a metastable peptide trapped inside the $\alpha$-HL's at negative $\Delta V$ s exposes its Arg residues to the nanopore's lumen. Consequently, this may render the volumetric difference readout between Ala and Trp residues blocking the constriction region "hidden", by the large resistance contribution of the Arg tail in the lumen. Although we lack a strong proof of this, the indirect hint from our MD simulations shows that when the lumen and the constriction are fully occupied by large residues such as Arg and Trp, we have a similar current blockade (Figure 4, panel d).

It remains an open question regarding a solution enabling unidirectional, slowed down movement across the nanopore of a denaturated, heterogeneously charged polypeptide, enabling the unequivocal statistical description of the translocation process at the constriction region of the nanopore and discrimination of all individual residues. This technique may become, however, a stepping stone for more refined approaches to probe the structure of specific peptide domains containing patches of neutral residues, pinpoint existing mutations or physicochemical alterations of neutral amino acid patches, or specific post-translational modifications. It may provide a sensitive approach to discriminate among overall neutral amino acid enantiomers on a specific peptide domain.

\section{ASSOCIATED CONTENT}

\section{S Supporting Information}

The Supporting Information is available free of charge on the ACS Publications website at DOI: 10.1021/acs.langmuir.7b03163. 
Additional data analysis and $\mathrm{MD}$ simulations (PDF)

\section{AUTHOR INFORMATION}

\section{Corresponding Authors}

*E-mail: mauro.chinappi@uniroma2.it.

*E-mail: y_k_park@chosun.ac.kr.

*E-mail: luchian@uaic.ro.

\section{ORCID}

Tudor Luchian: 0000-0002-9388-7266

\section{Author Contributions}

${ }^{\mathrm{I}}$ A.A. and A.E.R. contributed equally. T.L. and Y.P. conceived the idea, A.A. contributed to the experimental part, data processing and analysis, A.E.R. and M.C. contributed to the molecular simulations and data analysis, T.L. and Y.P. contributed to the data analysis and acquisition of funding. All authors contributed to the manuscript draft, and have given approval to the final version of the manuscript.

\section{Notes}

The authors declare no competing financial interest.

\section{ACKNOWLEDGMENTS}

The authors acknowledge the financial support offered by the National Research Foundation of Korea (NRF) funded by Grant no. NRF-2014K1A1A2064460 and 2016R1A2A1A05005440, the Global Collaborative R\&D program (N0001229), and Grant no. PN-III-P4-ID-PCE2016-0026 (NANOTWEEZ).

\section{REFERENCES}

(1) Kasianowicz, J. J.; Robertson, J. W. F.; Chan, E. R.; Reiner, J. E.; Stanford, V. M. Nanoscopic Porous Sensors. Annu. Rev. Anal. Chem. 2008, 1, 737-766.

(2) Bayley, H. Nanopore Sequencing: from Imagination to Reality. Clin. Chem. 2015, 61, 25-31.

(3) Muthukumar, M.; Plesa, C.; Dekker, C. Single-Molecule Sensing with Nanopores. Phys. Today 2015, 68, 40-46.

(4) Kasianowicz, J. J.; Brandin, E.; Branton, D.; Deamer, D. W. Characterization of Individual Polynucleotide Molecules Using a Membrane Channel. Proc. Natl. Acad. Sci. U. S. A. 1996, 93, 1377013773.

(5) Branton, D.; Deamer, D. W.; Marziali, A.; Bayley, H.; Benner, S. A.; Butler, T.; Di Ventra, M.; Garaj, S.; Hibbs, A.; Huang, X.; Jovanovich, S. B.; Krstic, P. S.; Lindsay, S.; Ling, X. S.; Mastrangelo, C. H.; Meller, A.; Oliver, J. S.; Pershin, Y. V.; Ramsey, J. M.; Riehn, R.; Soni, G. V.; Tabard-Cossa, V.; Wanunu, M.; Wiggin, M.; Schloss, J. A. The Potential and Challenges of Nanopore Sequencing. Nat. Biotechnol. 2008, 26, 1146-1153.

(6) Ashkenasy, N.; Sánchez-Quesada, J.; Bayley, H.; Ghadiri, M. R. Recognizing a Single Base in an Individual DNA Strand: A Step toward Nanopore DNA Sequencing. Angew. Chem., Int. Ed. 2005, 44, 1401-1404.

(7) Akeson, M.; Branton, D.; Kasianowicz, J. J.; Brandin, E.; Deamer, D. W. Microsecond Time-Scale Discrimination among Polycytidylic Acid, Polyadenylic Acid, and Polyuridylic Acid as Homopolymers or as Segments within Single RNA Molecules. Biophys. J. 1999, 77, 32273233.

(8) Meller, A.; Nivon, L.; Brandin, E.; Golovchenko, J.; Branton, D. Rapid Nanopore Discrimination between Single Polynucleotide Molecules. Proc. Natl. Acad. Sci. U. S. A. 2000, 97, 1079-1084.

(9) Venkatesan, B. M.; Bashir, R. Nanopore Sensors for Nucleic Acid Analysis. Nat. Nanotechnol. 2011, 6, 615-624.

(10) Cao, C.; Ying, Y.-L.; Hu, Z.-L.; Liao, D.-F.; Tian, H.; Long, Y.-T. Discrimination of Oligonucleotides of Different Lengths with a WildType Aerolysin Nanopore. Nat. Nanotechnol. 2016, 11, 713-718.
(11) Movileanu, L.; Schmittschmitt, J.; Scholtz, J. M.; Bayley, H. Interaction of Peptides with a Protein Nanopore. Biophys. J. 2005, 89, $1030-1045$.

(12) Nivala, J.; Marks, D. B.; Akeson, M. Unfoldase-Mediated Protein Translocation through an $\alpha$-Hemolysin Nanopore. Nat. Biotechnol. 2013, 31, 247-250.

(13) Oukhaled, A.; Bacri, L.; Pastoriza-Gallego, M.; Betton, J. M.; Pelta, J. Sensing Proteins through Nanopores: Fundamental to Applications. ACS Chem. Biol. 2012, 7, 1935-1949.

(14) Pastoriza-Gallego, M.; Rabah, L.; Gibrat, G.; Thiebot, B.; van der Goot, F. G.; Auvray, L.; Betton, J.-M.; Pelta, J. Dynamics of Unfolded Protein Transport through an Aerolysin Pore. J. Am. Chem. Soc. 2011, 133, 2923-2931.

(15) Rodriguez-Larrea, D.; Bayley, H. Multistep Protein Unfolding during Nanopore Translocation. Nat. Nanotechnol. 2013, 8, 288-295.

(16) Talaga, D. S.; Li, J. Single-Molecule Protein Unfolding in Solid State Nanopores. J. Am. Chem. Soc. 2009, 131, 9287-9297.

(17) Mereuta, L.; Asandei, A.; Seo, C. H.; Park, Y.; Luchian, T. Quantitative Understanding of $\mathrm{pH}$ - and Salt-Mediated Conformational Folding of Histidine-Containing, $\beta$-Hairpin-Like Peptides, through Single-Molecule Probing with Protein Nanopores. ACS Appl. Mater. Interfaces 2014, 6, 13242-13256.

(18) Bonome, E. L.; Lepore, R.; Raimondo, D.; Cecconi, F.; Tramontano, A.; Chinappi, M. Multistep Current Signal in Protein Translocation through Graphene Nanopores. J. Phys. Chem. B 2015, $119,5815-5823$.

(19) Rosen, C. B.; Rodriguez-Larrea, D.; Bayley, H. Single-Molecule Site-Specific Detection of Protein Phosphorylation with a Nanopore. Nat. Biotechnol. 2014, 32, 179-181.

(20) Wilson, J.; Sloman, L.; He, Z.; Aksimentiev, A. Graphene Nanopores for Protein Sequencing. Adv. Funct. Mater. 2016, 26, $4830-4838$.

(21) Song, L.; Hobaugh, M. R.; Shustak, C.; Cheley, S.; Bayley, H.; Gouaux, J. E. Structure of Staphylococcal Alpha-Hemolysin, a Heptameric Transmembrane Pore. Science 1996, 274, 1859-1866.

(22) Aksimentiev, A.; Schulten, K. Imaging $\alpha$-Hemolysin with Molecular Dynamics: Ionic Conductance, Osmotic Permeability, and the Electrostatic Potential Map. Biophys. J. 2005, 88, 3745-3761.

(23) Faller, M.; Niederweis, M.; Schulz, G. E. The Structure of a Mycobacterial Outer-Membrane Channel. Science 2004, 303, 11891192.

(24) Derrington, I. M.; Butler, T. Z.; Collins, M. D.; Manrao, E.; Pavlenok, M.; Niederweis, M.; Gundlach, J. H. Nanopore DNA Sequencing with MspA. Proc. Natl. Acad. Sci. U. S. A. 2010, 107, 16060-16065.

(25) Lu, B.; Fleming, S.; Golovchenko, J. Thermal Motion of DNA in an MspA Pore. Biophys. J. 2015, 109, 1439-1445.

(26) Fologea, D.; Uplinger, J.; Thomas, B.; McNabb, D. S.; Li, J. Slowing DNA Translocation in a Solid-State Nanopore. Nano Lett. 2005, 5, 1734-1737.

(27) Kowalczyk, S. W.; Wells, D. B.; Aksimentiev, A.; Dekker, C. Slowing Down DNA Translocation through a Nanopore in Lithium Chloride. Nano Lett. 2012, 12, 1038-1044.

(28) Angevine, C. E.; Chavis, A. E.; Kothalawala, N.; Dass, A.; Reiner, J. E. Enhanced single molecule mass spectrometry via charged metallic clusters. Anal. Chem. 2014, 86, 11077-11085.

(29) Ying, Y.-L.; Li, D.-W.; Li, Y.; Lee, J. S.; Long, Y.-T. Enhanced Translocation of poly (dt) 45 through an $\alpha$-Hemolysin Nanopore by Binding with Antibody. Chem. Commun. 2011, 47, 5690-5692.

(30) Schiel, M.; Siwy, Z. S. Diffusion and Trapping of Single Particles in Pores with Combined Pressure and Dynamic Voltage. J. Phys. Chem. C 2014, 118, 19214-19223.

(31) Di Fiori, N.; Squires, A.; Bar, D.; Gilboa, T.; Moustakas, T. D.; Meller, A. Optoelectronic Control of Surface Charge and Translocation Dynamics in Solid-State Nanopores. Nat. Nanotechnol. 2013, $8,946-951$.

(32) Firnkes, M.; Pedone, D.; Knezevic, J.; Doblinger, M.; Rant, U. Electrically Facilitated Translocations of Proteins through Silicon 
Nitride Nanopores: Conjoint and Competitive Action of Diffusion, Electrophoresis, and Electroosmosis. Nano Lett. 2010, 10, 2162-2167.

(33) Mereuta, L.; Roy, M.; Asandei, A.; Lee, J. K.; Park, Y.; Andricioaei, I.; Luchian, T. Slowing Down Single-Molecule Trafficking through a Protein Nanopore Reveals Intermediates for Peptide Translocation. Sci. Rep. 2015, 4, 3885.

(34) Asandei, A.; Schiopu, I.; Chinappi, M.; Seo, C. H.; Park, Y.; Luchian, T. Electroosmotic Trap Against the Electrophoretic Force Near a Protein Nanopore Reveals Peptide Dynamics During Capture and Translocation. ACS Appl. Mater. Interfaces 2016, 8, 13166-13179.

(35) Schmitt-Kopplin, P.; Frommberger, M. Capillary Electrophoresis-Mass Spectrometry: 15 Years of Developments and Applications. Electrophoresis 2003, 24, 3837-3867.

(36) Laursen, R. A. Solid-Phase Edman Degradation. Eur. J. Biochem. 1971, 20, 89-102.

(37) Kennedy, E.; Dong, Z.; Tennant, C.; Timp, G. Reading the Primary Structure of a Protein with $0.07 \mathrm{~nm} 3$ Resolution Using a Subnanometre-Diameter Pore. Nat. Nanotechnol. 2016, 11, 968-976.

(38) Boynton, P.; Di Ventra, M. Sequencing Proteins with Transverse Ionic Transport in Nanochannels. Sci. Rep. 2016, 6, 25232.

(39) Ohshiro, T.; Tsutsui, M.; Yokota, K.; Furuhashi, M.; Taniguchi, M.; Kawai, T. Detection of Post-Translational Modifications in Single Peptides Using Electron Tunnelling Currents. Nat. Nanotechnol. 2014, 9, 835-840.

(40) Zhao, Y.; Ashcroft, B.; Zhang, P.; Liu, H.; Sen, S.; Song, W.; Im, J.; Gyarfas, B.; Manna, S.; Biswas, S.; Borges, C.; Lindsay, S. SingleMolecule Spectroscopy of Amino Acids and Peptides by Recognition Tunneling. Nat. Nanotechnol. 2014, 9, 466-473.

(41) Wloka, C.; Van Meervelt, V.; van Gelder, D.; Danda, N.; Jager, N.; Williams, C. P.; Maglia, G. Label-Free and Real-Time Detection of Protein Ubiquitination with a Biological Nanopore. ACS Nano 2017, $11,4387-4394$.

(42) Montal, M.; Mueller, P. Formation of Bimolecular Membranes from Lipid Monolayers and a Study of their Electrical Properties. Proc. Natl. Acad. Sci. U. S. A. 1972, 69, 3561-3566.

(43) Asandei, A.; Apetrei, A.; Park, Y.; Hahm, K. S.; Luchian, T. Investigation of Single-Molecule Kinetics Mediated by Weak Hydrogen-Bonds within a Biological Nanopore. Langmuir 2011, 27, 19-24.

(44) Phillips, J. C.; Braun, R.; Wang, W.; Gumbart, J.; Tajkhorshid, E.; Villa, E.; Chipot, C.; Skeel, R. D.; Kalé, L.; Schulten, K. Scalable molecular dynamics with namd. J. Comput. Chem. 2005, 26, 17811802 .

(45) Brooks, B. R.; Brooks, C. L., 3rd; Mackerell, A. D., Jr.; Nilsson, L.; Petrella, R. J.; Roux, B.; Won, Y.; Archontis, G.; Bartels, C.; Boresch, S.; Caflisch, A.; Caves, L.; Cui, Q.; Dinner, A. R.; Feig, M.; Fischer, S.; Gao, J.; Hodoscek, M.; Im, W.; Kuczera, K.; Lazaridis, T.; Ma, J.; Ovchinnikov, V.; Paci, E.; Pastor, R. W.; Post, C. B.; Pu, J. Z.; Schaefer, M.; Tidor, B.; Venable, R. M.; Woodcock, H. L.; Wu, X.; Yang, W.; York, D. M.; Karplus, M. Charmm: the Biomolecular Simulation Program. J. Comput. Chem. 2009, 30, 1545-1614.

(46) Jorgensen, W. L.; Chandrasekhar, J.; Madura, J. D.; Impey, R. W.; Klein, M. L. Comparison of Simple Potential Functions for Simulating Liquid Water. J. Chem. Phys. 1983, 79, 926-935.

(47) Luo, Y.; Roux, B. Simulation of Osmotic Pressure in Concentrated Aqueous Salt Solutions. J. Phys. Chem. Lett. 2010, 1, $183-189$.

(48) Comer, J. R.; Wells, D. B.; Aksimentiev, A. Modeling Nanopores for Sequencing DNA. Methods Mol. Biol. 2011, 749, 317-358.

(49) Bonome, E. L.; Cecconi, F.; Chinappi, M. Electroosmotic Flow through an Alpha-Hemolysin Nanopore. Microfluid. Nanofluid. 2017, $21,96$.

(50) Lomize, M. A.; Lomize, A. L.; Pogozheva, I. D.; Mosberg, H. I. OPM: Orientations of Proteins in Membranes Database. Bioinformatics 2006, 22, 623-625.

(51) Humphrey, W.; Dalke, A.; Schulten, K. Vmd: Visual Molecular Dynamics. J. Mol. Graphics 1996, 14, 33-38.

(52) Thévenet, P.; Shen, Y.; Maupetit, J.; Guyon, F.; Derreumaux, P.; Tufféry, P. PEP-FOLD: an Updated de Novo Structure Prediction
Server for Both Linear and Disulfide Bonded Cyclic Peptides. Nucleic Acids Res. 2012, 40, W288-W293.

(53) Gumbart, J.; Khalili-Araghi, F.; Sotomayor, M.; Roux, B. Constant Electric Field Simulations of the Membrane Potential Illustrated with Simple Systems. Biochim. Biophys. Acta, Biomembr. 2012, 1818, 294-302.

(54) Asandei, A.; Chinappi, M.; Lee, J.-K.; Chang, H. S.; Mereuta, L.; Park, Y.; Luchian, T. Placement of Oppositely Charged Aminoacids at a Polypeptide Termini Determines the Voltage-Controlled Braking of Polymer Transport through Nanometer-Scale Pores. Sci. Rep. 2015, 5, 10419.

(55) Asandei, A.; Chinappi, M.; Kang, H.-K.; Seo, C. H.; Mereuta, L.; Park, Y.; Luchian, T. Acidity-Mediated, Electrostatic Tuning of Asymmetrically Charged Peptides Interactions with Protein Nanopores. ACS Appl. Mater. Interfaces 2015, 7, 16706-16714.

(56) Chinappi, M.; Luchian, T.; Cecconi, F. Nanopore Tweezers: Voltage Controlled Trapping and Releasing of Analytes. Phys. Rev. E 2015, 92, 032714.

(57) Mathé, J.; Aksimentiev, A.; Nelson, D. R.; Schulten, K.; Meller, A. Orientation Discrimination of Single-Stranded DNA Inside the $\alpha$ Hemolysin Membrane Channel. Proc. Natl. Acad. Sci. U. S. A. 2005, $102,12377-12382$. 